\begin{tabular}{|c|c|}
\hline Title & A photon position sensor consisting of single electron circuits \\
\hline Author(s) & Kikombo, A ndrew Kilinga; Tabe, Michiharu; A memiya, Y oshihito \\
\hline Citation & $\begin{array}{l}\text { Nanotechnology, 20(40), 405209 } \\
\text { https://doi.org/10.1088/0957-4484/20/40/405209 }\end{array}$ \\
\hline Issue Date & 2009-10-07 \\
\hline Doc URL & http:/hdl. handle.net/2115/39620 \\
\hline Rights & $\begin{array}{l}\text { This is an author-created, un-copyedited version of an article accepted for publication in Nanotechnology. IOP } \\
\text { Publishing L td is not responsible for any errors or omissions in this version of the manuscript or any version derived } \\
\text { from it. The definitive publisher authenticated version is avail able online at } 10.1088 / 0957-4484 / 20 / 40 / 405209\end{array}$ \\
\hline Type & article (author version) \\
\hline File Information & NT20-40_405209.pdf \\
\hline
\end{tabular}

Instructions for use 


\title{
Photon position sensor consisting of single-electron circuits
}

\author{
Andrew Kilinga Kikombo ${ }^{1}$, Michiharu Tabe ${ }^{2}$, and Yoshihito \\ Amemiya $^{1}$ \\ ${ }^{1}$ Department of Electrical Engineering, Hokkaido University, Sapporo, 060-8628 \\ Japan \\ 2 Research Institute of Electronics, Shizuoka University, Japan. \\ E-mail: kikombo@sapiens-ei.eng.hokudai.ac.jp
}

\begin{abstract}
This paper proposes a solid-state sensor that can detect the position of incident photons with a high spatial resolution. The sensor consists of a twodimensional array of single-electron oscillators, each coupled to its neighbors through coupling capacitors. An incident photon triggers an excitatory circular wave of electron tunneling in the oscillator array. The wave propagates in all directions to reach the periphery of the array. By measuring the arrival time of the wave at the periphery, we can know the position of the incident photon. Tunneling wave's generation, propagation, arrival at the array periphery, and the determination of incident photon positions are demonstrated with the results of Monte-Carlo based computer simulations.
\end{abstract}

PACS numbers: 85.35

\section{Introduction}

A promising area of research in microphotonics is the development of solid-state sensors that can detect the position of incident single photons with high spatial resolution. This paper presents one such device, a two-dimensional photon sensor consisting of singleelectron integrated circuits.

Detecting the position of incident photons is a fundamental, important process in spectroscopic and imaging measurement in physical, chemical, and biological sciences. The existing device used for this purpose is the microchannel plate photomultiplier (MCP) - a two-dimensional array of millions of small photomultiplier tubes, each tube with a diameter of several micrometers. The MCP is a superior device with a high sensitivity and a large signal-to-noise ratio. Its spatial resolving power, however, is limited to the pitch of the photomultiplier tube array and difficult to set to less than 10 $\mu \mathrm{m}$.

Far higher resolution can be achieved using an array of single-electron tunneling junctions. A single-electron tunneling junction can operate as a minute photon detector based on the photo-induced charging effect (see [1] and [2] for details). Therefore, 


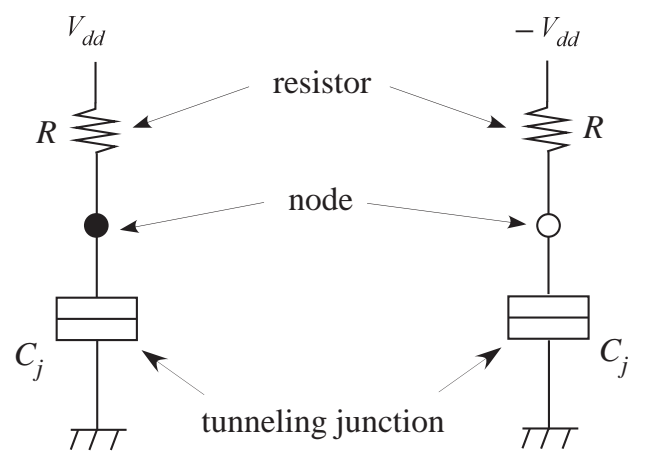

(i) (ii)

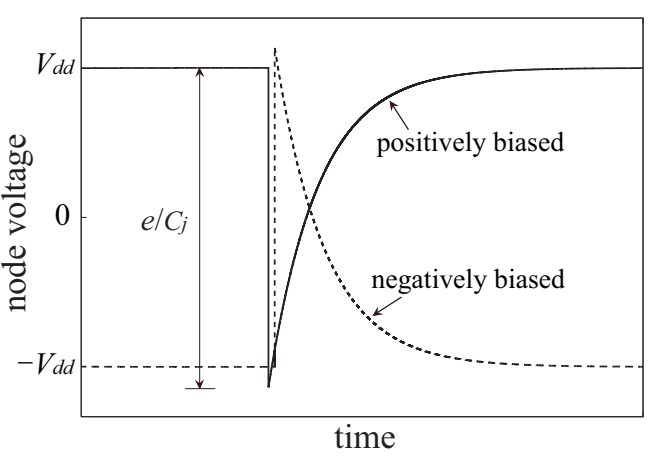

(b)$$
\text { ) }
$$

(a)

Figure 1. Single electron oscillators biased with (a-(i)) positive voltage $V_{d d}$ and (a(ii)) negative voltage $-V_{d d}$, and (b) monostable, or one-shot, operation of positively biased (solid line) and negatively biased (dashed line) oscillators.

arranging many tunneling junctions into an array will create a novel two-dimensional position sensor for photons. Junction arrays with a tens-nanometer arrangement pitch can be fabricated using existing nanoprocess technologies, so one can develop a position sensor with a high resolving power of 0.01-0.1 $\mu \mathrm{m}-\mathrm{a}$ far higher resolution than that of MCPs.

The challenge in the development of this sensor is related to the accurate determination of the two-dimensional position of incident photons. Unlike conventional devices, it is difficult to lay row-column access lines through closely arranged, minute tunneling junctions. Even if one could do that, the large parasitic capacitance of the access lines would impede the single-electron operation of the tunneling junctions.

To solve this problem, we developed a method of detecting positions that makes use of the propagation of tunneling waves in a tunneling junction array. The proposed photon sensor consists of many single-electron tunneling oscillators regularly arrayed on a plane, where each oscillator is coupled with neighboring oscillators. If a photon hits and excites an oscillator, photo-induced tunneling occurs in the oscillator. This induces tunneling in neighboring junctions, and the tunneling events successively propagate, like a wave, in all directions to reach the periphery of the sensor. By measuring the arrival time of the wave at several points on the periphery, one can determine the starting point of the wave and therefore know the position at which the photon entered the sensor.

In the following sections, first the structure of the proposed sensor is described. Then its operation is illustrated using the results of Monte-Carlo based computer simulation (See appendix A for the method of simulation). Tunneling wave's generation, propagation, and arrival at the device periphery are simulated. We show that the starting position of the wave can be determined using the arrival time of the wave. 


\section{Structure of sensor device}

To make the photon position sensor, we use a network of single-electron oscillators. A single-electron oscillator, shown in Fig. 1, is a simple circuit consisting of a tunneling junction $C_{j}$ and a high-resistance resistor $R$ connected in series at a node and biased with a positive voltage $V_{d d}$ (Fig. $\left.1(\mathrm{a}-(\mathrm{i}))\right)$ or a negative voltage $-V_{d d}$ (Fig. 1(a-(ii))). It operates as a relaxation oscillator at low temperatures at which the Coulomb-blockade effect occurs. The oscillator is astable if $V_{d d}>e /\left(2 C_{j}\right)$ ( $e$ is the elementary charge) and monostable if $V_{d d}<e /\left(2 C_{j}\right)$ (see [3] and [4] for detailed explanation). The node voltage of the monostable oscillator is equal to the bias voltage in an equilibrium state, and no change occurs under that condition. Upon application of a triggering signal, such as an incidence of photons, the Coulomb blockade is broken off, and electron tunneling occurs through the tunneling junctio $\mathrm{n}$. The node voltage of a positively biased oscillator drops by $e / C_{j}$, as shown in Fig. 1(b), because of tunneling from the ground to the node. The node voltage then gradually increases to return to $V_{d d}$ as junction capacitance $C_{j}$ is charged through resistance $R$. In a negatively biased oscillator, the node voltage jumps by $e / C_{j}$ because of tunneling from the node to the ground, and then gradually decreases to return to $-V_{d d}$.

The structure of the proposed sensor consisting of monostable oscillators is shown in Fig. 2. It consists of a network of monostable single-electron oscillators-positively biased and negatively biased oscillators - regularly arrayed on a plane in a checkered pattern. Each positively biased oscillator is connected to four negatively biased oscillators by means of coupling capacitors. Similarly, each negatively biased oscillator is connected to four positively biased oscillators. The unit length of the network, or the arrangement pitch of oscillators, could be tens of nanometers for tunneling junction arrays that are made using existing nanoprocess technologies. The network, or the sensor device, is in a stable uniform state as it stands. Once tunneling is induced in an oscillator by an incident photon, the occurrence of tunneling events is transmitted from the oscillator to other oscillators in the following manner (see [5] for details). For example, if electron tunneling occurs in a positively biased oscillator, the oscillator changes its node voltage from positive to negative, and this induces, through the coupling capacitors, electron tunneling in neighboring negatively biased oscillators. The negatively biased oscillators consequently change their node voltages from negative to positive, and this induces electron tunneling in neighboring positively biased oscillators. In this manner, the occurrence of tunneling events is transmitted in the network like a wave-hereafter, this consecutive transfer of tunneling events (wave) is referred to as a tunneling wave.

To show this operation simply, the transmission of a tunneling wave is illustrated with simulated results for a one-dimensional connection of oscillators given in Fig. 3(a). Electron tunneling was induced in the leftmost oscillator A0 by a triggering signal, and the chain reaction of tunneling started and proceeded to the right along the row of oscillators. The successive drops in the node voltages, shown in Figs. 3(b) and (c), indicate this chain reaction, which occurred in the following manner. When tunneling occurred in the positively biased oscillator A2, its node voltage changed from positive 


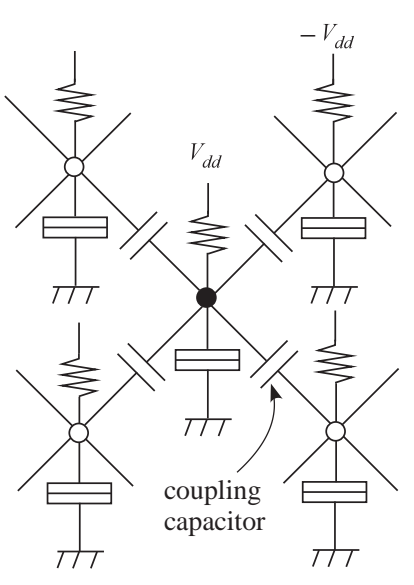

(a)

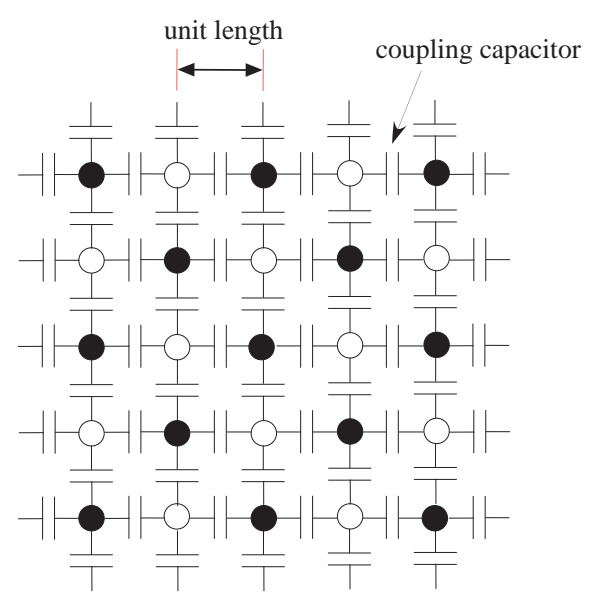

(b)

Figure 2. Photon-position sensor consisting of two-dimensional network of singleelectron oscillators: (a) capacitive coupling of positively biased and negatively biased oscillators and (b) sensor device consisting of coupled-oscillator network. Closed circles and open circles represent the node of positively biased and negatively biased oscillators.

to negative (see Fig. 3(b)). This induced tunneling in the neighboring negatively biased oscillator A3. The node voltage of A3 therefore changed from negative to positive, and this induced tunneling in positively biased oscillator A4. This way, a tunneling wave could propagate rightward through the oscillators.

A time lag or a waiting time exists between when the voltage across each junction exceeds the tunneling threshold and when tunneling actually occurs in the junction. This is caused by the stochastic nature of tunneling and determines the velocity of wave propagation. The waiting time has probabilistic fluctuations in every tunneling event (e.g., see the difference between $t_{1}, t_{2}$, and $t_{3}$ in Fig. 3(b)), and consequently, the wave velocity fluctuates at every moment around its mean value. The effect of this fluctuation, however, diminishes as the number of oscillators increases (see Appendix B).

\section{Tunneling waves propagating in the oscillator network}

In the two-dimensional network (Fig. 2(b)), the tunneling wave propagates in all direction and spreads across the network to form a circular wave. We simulated the wave propagation in a network consisting of $501 \times 501$ positively biased oscillators interleaved with a $500 \times 500$ array of negatively biased oscillators in a checkerboard pattern. In the calculations, we assumed that each oscillator is connected to four neighboring oscillators inside the network, three oscillators on the four sides, and two oscillators at the four corners.

The result is shown in Fig. 4. To visualize the time development of a tunneling wave, 


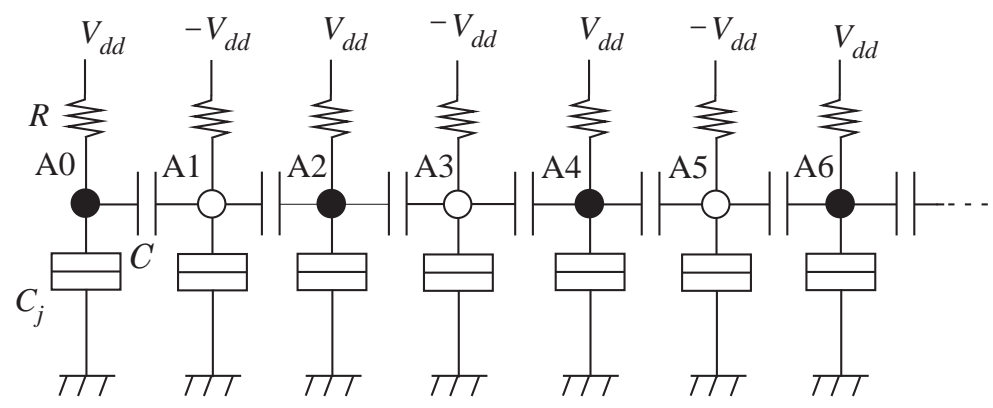

(a)

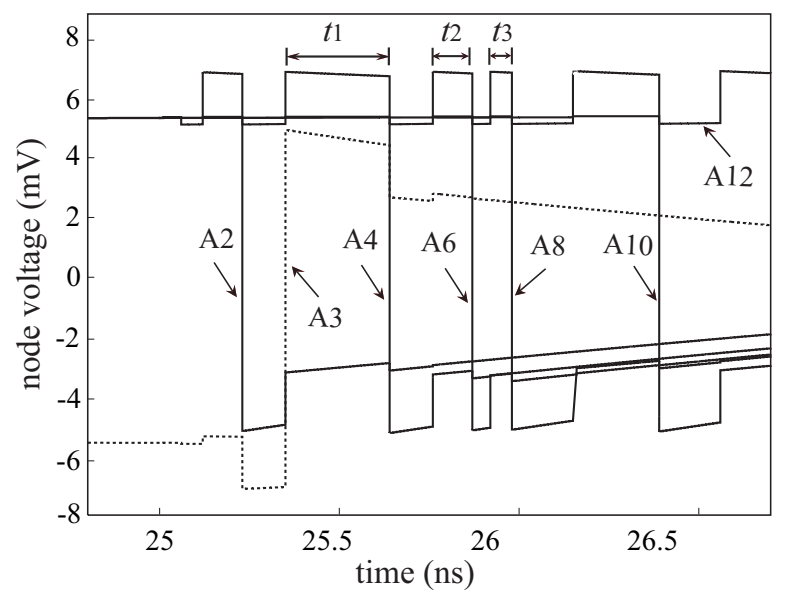

(b)

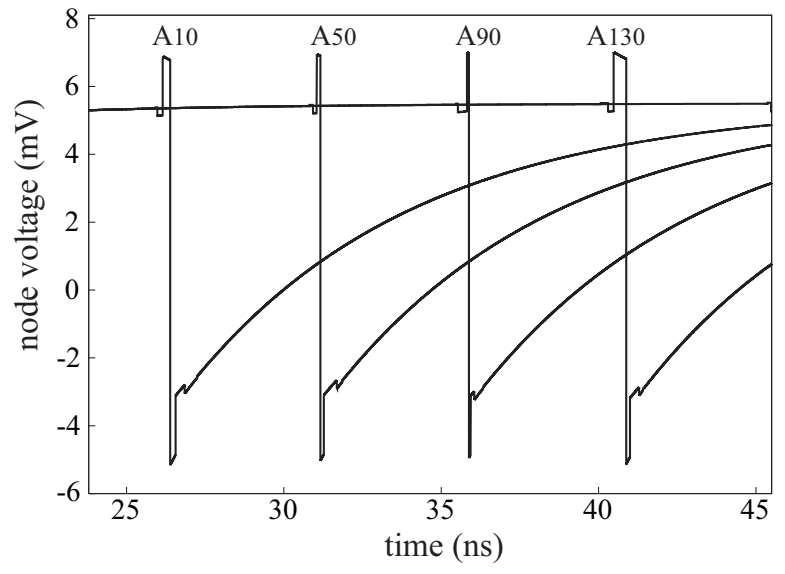

(c)

Figure 3. (a) One-dimensional connection of oscillators A0, A1, A2, ...; (b) Waveform of node voltage plotted for positively biased oscillators A2-A12 (solid lines) and negatively biased oscillator A3 (dashed line); (c) waveform for every fortieth oscillator. Tunneling is induced in leftmost oscillator A0 and transmitted rightward along the row of oscillators with delay. Drop in each waveform corresponds to tunneling in the oscillator. This was simulated with a set of parameters $C_{j}=10 \mathrm{aF}, C=2 \mathrm{aF}, R=$ $400 \mathrm{M} \Omega$, tunneling junction conductance $=1 \mu \mathrm{S}, V_{d d}=5.5 \mathrm{mV},-V_{d d}=-5.5 \mathrm{mV}$, and zero temperature. 


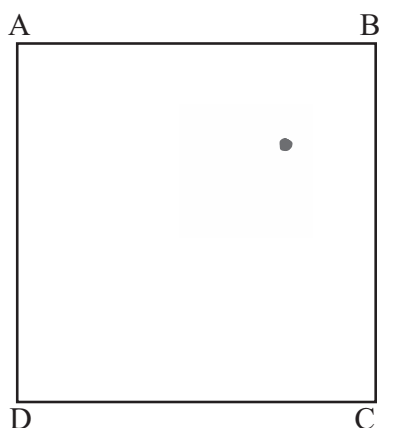

(a) $t=3 \mathrm{~ns}$

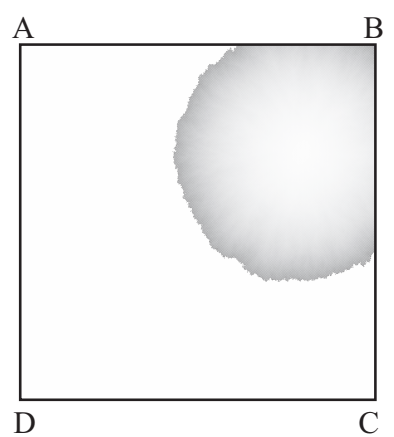

(d) $t=45 \mathrm{~ns}$

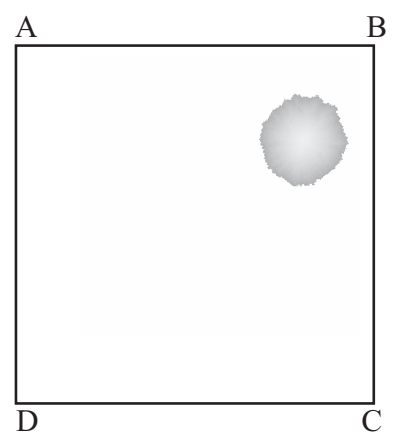

(b) $t=10 \mathrm{~ns}$

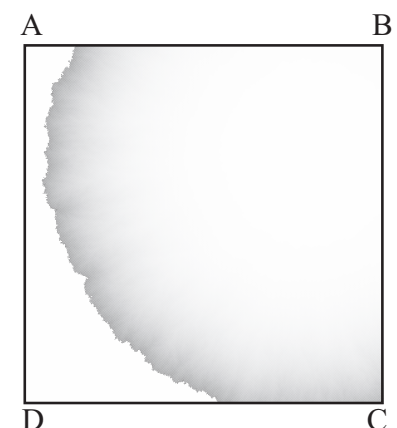

(e) $t=90 \mathrm{~ns}$

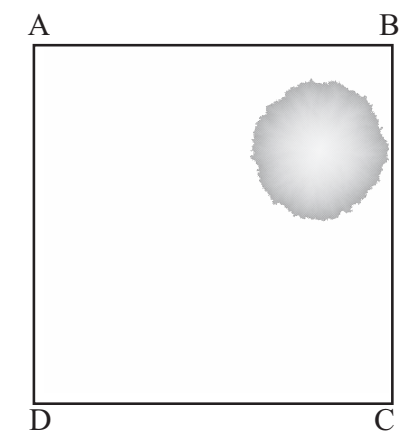

(c) $t=25 \mathrm{~ns}$

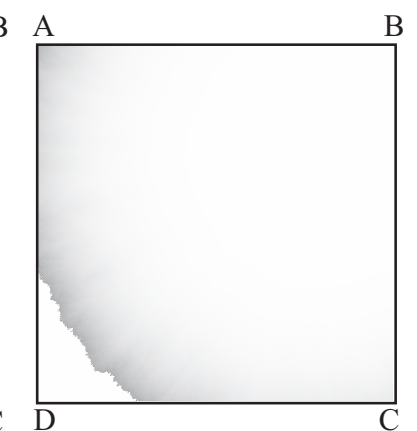

(f) $t=105 \mathrm{~ns}$

Figure 4. Expanding circular wave in the network. Snapshots for six time steps, with time $t$ after the start of the wave, simulated with a set of parameters $C_{j}=10 \mathrm{aF}, C$ $=2 \mathrm{aF}, R=400 \mathrm{M} \Omega$, tunneling junction conductance $=1 \mu \mathrm{S}, V_{d d}=4.8 \mathrm{mV},-V_{d d}=$ $-4.8 \mathrm{mV}$, and zero temperature.

the node voltage of each positively biased oscillator is shown on a gray scale: the light shading represents high voltage, and the dark represents low voltage. In this example, a tunneling wave started at the upper right of the network (Fig. 4(a)), expanded in all directions in the form of a circular wave (Figs. 4(b) and (c)), and reached corners A, B, C, and D (Figs. 4(d)-(f)). The front of the wave is the region where tunneling just occurred, and therefore, the node voltages of oscillators are at the lowest negative value (represented by the darkest shading). The front line of the wave is uneven or irregular because the velocity of the traveling wave fluctuated in each direction throughout the process because of the stochastic waiting time of tunneling. The mean velocity depends on the circuit parameters and was 100 unit lengths per $12.52 \mathrm{~ns}$ (for 'unit leng th', see Fig. 2(b)) for the parameter set given in the figure caption.

On the arrival of the wave, the positively biased oscillator at each corner produced tunneling and changed its node voltage from positive to negative. Waveforms of the node voltages are plotted in Fig. 5 - the results of the wave propagation shown in Fig. 4. The time when the voltage dropped indicates the time when the wave reached the corner of the network.

After the wave passed, the node voltage of each oscillator gradually increased to return to its initial value. This is represented by light shading behind the wave front. 


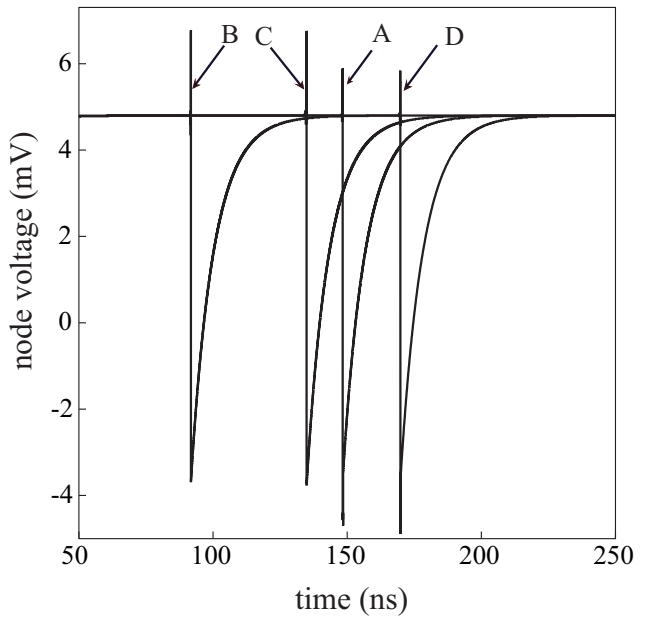

Figure 5. Node voltage waveforms of the oscillators at corners A, B, C, and D of network: the result of the wave propagation shown in Fig. 4. The trigger was applied at time $=50 \mathrm{~ns}$

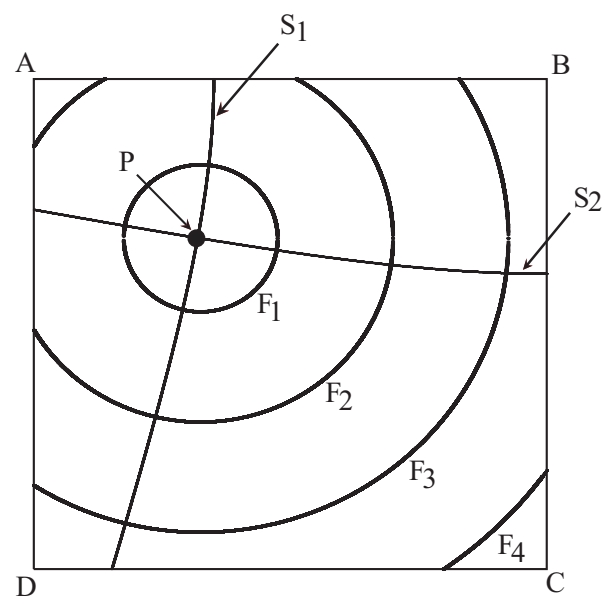

Figure 6. Determination of the position of incident photons. ABCD: sensor network. P: incident position of a photon, F1-F4: spreading wave front for four steps. S1 and S2: hyperbolas drawn from the arrival-time data at points A, $\mathrm{B}$, and $\mathrm{C}$.

If a photon hits again, the same operation will be repeated.

\section{Detecting the starting position of tunneling waves}

From the wave's arrival time at the corners, we can know the starting position of the wave as follows (see Fig. 6). Let us assume that a photon hits the sensor network ABCD at a position $\mathrm{P}$. Then a tunneling wave appeares at $\mathrm{P}$ and spreads out through the sensor at a velocity of $v_{0}$. As time passes, the wave front expands in all directions, as indicated by F1-F4, and reaches the periphery of the sensor. We observe the arrival of the wave at corners A, B, C, and D and measure the wave's arrival time at each point. If the arrival time was $t_{0}$ at point $\mathrm{A}$ and $t_{0}+t_{1}$ at point $\mathrm{B}$, we can consider that position $\mathrm{P}$ is on the locus $\mathrm{S} 1$ of points where the difference in the distance to points $\mathrm{A}$ and $\mathrm{B}$ is $v_{0} t_{1}$. That is, $\mathrm{P}$ is on hyperbola $\mathrm{S} 1$ with foci $\mathrm{A}$ and $\mathrm{B}$. Similarly, position $\mathrm{P}$ is also on hyperbola $\mathrm{S} 2$ with foci $\mathrm{B}$ and $\mathrm{C}$. Therefore, we can determine position $\mathrm{P}$ as the point of intersection of the two hyperbolas.

There are six possible sets of foci $(\mathrm{AB}, \mathrm{AC}, \mathrm{AD}, \mathrm{BC}, \mathrm{BD}$, and $\mathrm{CD})$, so we can draw six hyperbolas from the data of arrival time at the four corner points. All the hyperbolas intersect at one point if the wave velocity is constant, and therefore, the two hyperbolas suffice to determine the position of P. In our device, however, wave velocity is not constant but fluctuates a little at every moment. Consequently, not all the hyperbolas intersect at one point, and there can be 15 intersection points at maximum (i.e., combination ${ }_{6} \mathrm{C}_{2}=15$ ). Therefore we determined the position of $\mathrm{P}$ by calculating the mean coordinate of the 15 intersection points. 


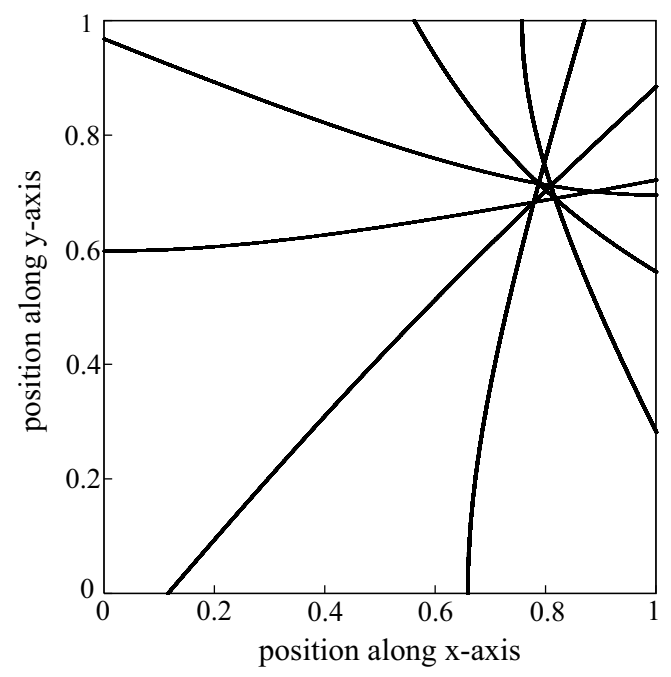

Figure 7. Calculation of the starting position of tunneling waves. The six hyperbolas with their 15 intersection points are shown. The mean coordinate of the intersection points was $(0.799,0.701)$, which was approximately consistent with the actual starting position $(0.8,0.7)$.

Taking the example of Fig. 4, we calculated the starting position of the wave from the data of the wave's arrival time given by Fig. 5, and compared the calculations with the actual starting position. In the calculations, we set the coordinates of corners $\mathrm{A}$, $\mathrm{B}, \mathrm{C}$, and $\mathrm{D}$ to $(0,1),(1,1),(1,0)$, and $(0,0)$. In this coordinate system, the starting position of the wave in Fig. 4 is $(0.8,0.7)$. In the calculation results, six hyperbolas with their 15 intersection points are shown in Fig. 7. The mean coordinate of the intersection points was $(0.799,0.701)$, which was approximately consistent with the actual starting position. This way, we can know the starting position of tunneling waves in the sensor, and therefore, can detect the position of incident photons.

\section{Toward actual devices}

To develop our idea into actual devices, we have to deal with two matters of importance; that is, constructing a large-scaled array of coupled single-electron oscillators, and amplifying a small output signal from a single-electron oscillator.

The key to constructing the device is forming the arrangement of single-electron oscillators with their coupling arms and tunneling junctions at an arrangement pitch of tens of nanometers. One of us previously presented and demonstrated a process technology that can be used to fabricate such an array structure [6]. This technology uses self-organized crystal growth based on selective-area metalorganic vapor-phase epitaxy (see [7] for this epitaxy method). Using this technology, we can form an array of semiconductor dots with coupling arms and tunneling junctions in a self-organizing manner. The next step is to create high-resistance resistors on the nanodots. This could be achieved by depositing a layer of high-resistive materials such as semi-insulating polycrystalline silicon (called SIPOS) [8]. 


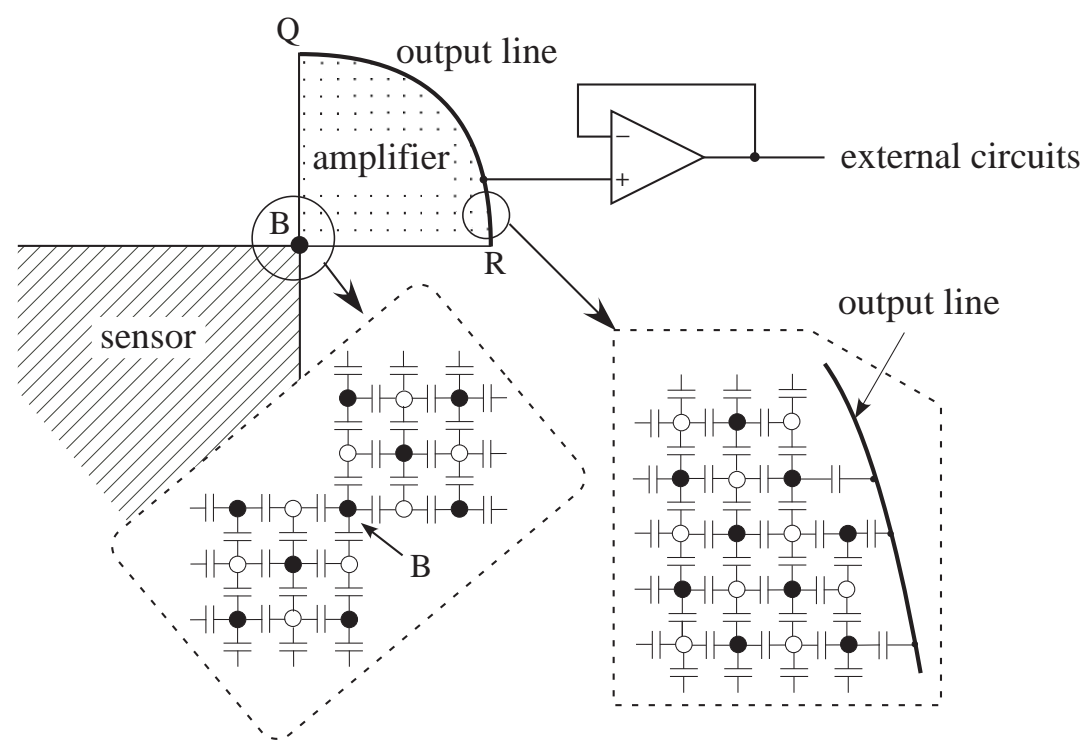

Figure 8. Output amplifier consisting of quadrant network of single-electron oscillators. Closed circles and open circles in the network represent the node of positively biased oscillators and negatively biased oscillators. The nodes of positively biased oscillators on arc QR are connected to an output load through coupling capacitors.

Amplifying the output signal is the other matter of importance. The arrival of a tunneling wave produces a change in the node voltage of the single-electron oscillator on each corner. However, we would not be able to retrieve the output signal directly from the corner oscillator. This is so because a single junction has a very small capacitance and therefore cannot send a large enough voltage signal to an output load that has a far larger capacitance. Therefore, an output amplifier is indispensable for efficiently driving the output load. A promising method of amplifying the single-electron signal is by making use of an expansion of tunneling waves in the single-electron network. A schematic diagram of such an amplifier is shown in Fig. 8. The amplifier consists of a single-electron network with a quadrant periphery BQR that is connected to corner oscillator B of the sensor. The amplifier network has the same structure as that shown in Fig. 2(b), and the nodes of the positively biased oscillators on arc QR are connected to an output line through capacitors. A tunneling wave generated in the sensing network reaches corner oscillator $\mathrm{B}$ and induces tunneling in the oscillator. This excites a secondary wave in the amplifier. The secondary wave expands in the amplifier network and reaches arc QR to induce tunneling in all oscillators connected to the output line. The oscillators have a large capacitance in total and therefore can send a large signal to an output load.

\section{Summary}

This paper proposed a photon position sensor with a high spatial resolving power. The sensor consists of monostable single-electron devices coupled with each other through 
capacitors. Through Monte-Carlo based computer simulations we confirmed that a tunneling wave can propagate through the network of oscillators toward the sensors positioned at the peripheries. From the arrival times at the peripheral sensors, we could accurately determine the starting position of the tunneling wave-hence the 2dimensional position where the incident photon hit the sensor. We are now developing the proposed sensor device with together with the amplifier, using the self-organized crystal growth process technology.

\section{Appendix A}

The following is an outline of the procedure of the Monte-Carlo simulation for single electron circuits used in this paper. This is in part an excerpt from [9]. Consider a circuit consisting of tunneling junctions (or tunneling capacitors), coupling capacitors, resistors, and biasing voltage sources. The internal state of the circuit is expressed by a set of charges on the nodes in the circuit. In the following, 'state' means this node-charge set. The voltage of each node can be calculated from the state.

To simulate the time-dependent behavior of the circuit, preset a time interval $\Delta t$ for iteration; $\Delta t$ is used to calculate the change in node charge caused by current flowing between bias voltages and nodes through resistors. The value of $\Delta t$ determines time resolution of simulation. A small $\Delta t$ increases the accuracy of simulation but requires a long computing time, resulting in a trade-off between the simulation time and precision. First, set time to 0 and give an initial charge to each node. Then simulate the circuit operation as follows.

Step 1: Update charge on each node. The updated charge $q_{i}$ for node $i$ is given by $q_{i}=q_{i 0}+\left(V_{b i}-V_{i}\right) \Delta t / R_{i}$, where $q_{i 0}$ is the node charge before updating, $V_{b i}$ is the bias voltage applied to node $i$ through resistance $R_{i}$, and $V_{i}$ is the voltage of node $i$. The state of the circuit changes from $\left(q_{10}, q_{20}, q_{30}, \ldots\right)$ to $\left(q_{1}, q_{2}, q_{3}, \ldots\right)$.

Step 2: Compute electrostatic energy $E_{0}$ (the sum of electrostatic energy of the tunneling junctions and coupling capacitors) for current state $\left(q_{1}, q_{2}, q_{3}, \ldots\right)$. Then enumerate all possible subsequent states and compute the electrostatic energy $E_{i}$ for each subsequent state $i$. (Subsequent state $i$ means a state into which the current state can be transformed by an electron tunneling between node $i$ and the ground. If the number of the tunnel junctions is $\mathrm{N}$, there are $2 \mathrm{~N}$ possible tunnelings and therefore $2 \mathrm{~N}$ subsequent states.)

Step 3: Compute the energy difference $\Delta E_{i}\left(=E_{0}-E_{i}\right)$ for each subsequent state. Using the value of $\Delta E_{i}$, calculate the waiting time for each tunneling event corresponding to each subsequent state. The waiting time $\tau_{i}$ is given by $\frac{1}{\Gamma_{i}} \ln \frac{1}{\gamma}$ where $\gamma$ is a uniform random number $(0<\gamma<1)$ generated for each tunneling event, and $\Gamma_{i}$ is the mean tunneling rate (the mean number of electrons that tunnel in one second) given by $\frac{\Delta E_{i}}{e^{2} R_{T} 1-\exp \left(-\Delta E_{i} / k_{B} T\right)}$, where $R_{T}$ is the tunneling resistance of the tunneling junction, $k_{B}$ is the Boltzmann constant, $e$ is the elementary charge, and $T$ is temperature.

Step 4: After calculating the waiting time $\tau_{i}$ for all possible tunnelings, take the shortest waiting time $\tau$, and compare it to $\Delta t$.

(i) If $\tau>\Delta t$, no tunneling occurs. Put time forward by $\Delta t$ and return to Step 1 to repeat the iteration. (ii) If $\tau \leq \Delta t$, the tunneling event corresponding to $\tau$ occurs. Cancel the operations in Step 1 and recalculate the updated charge on each node, using $\tau$ instead of $\Delta t$. The updated charge is given as $q_{i}=q_{i 0}+\left(V_{b i}-V_{i}\right) \tau / R_{i}-e$ for the node that receives an electron (tunneling) from the ground, or as $q_{i}=q_{i 0}+\left(V_{b i}-V_{i}\right) \tau / R_{i}+e$ for the node that sends an electron (tunneling) to the ground, and as $q_{i}=q_{i 0}+\left(V_{b i}-V_{i}\right) \tau / R_{i}$ for all the other nodes. Then, put time forward by $\tau$ and return to Step 1 to repeat the iterations. 


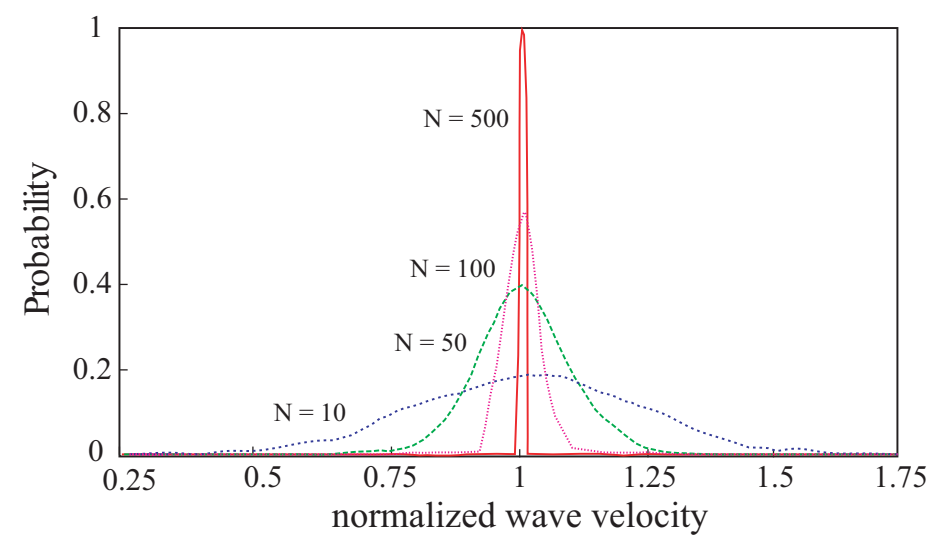

Figure 9. Fluctuation of wave velocity for $N$ (the number of oscillators) $=10,50$, 100, and 500. Horizontal axis shows the wave velocity (reciprocal of normalised mean waiting time). As $N$ increases, the fluctuation decreases and wave velocity approaches the average wave velocity.

\section{Appendix B}

The velocity of tunneling waves fluctuates around its mean value because of the stochastic nature of tunneling. However, when a wave travels across many oscillators, the fluctuation would be averaged and therefore the velocity could be considered to be almost constant. To confirm this, we simulated wave propagation along one-dimensional oscillator chains with various number of oscillators. That is, we simulated transmission time $T$ for a chain with $N$ oscillators and calculated the wave velocity given by $N / T$. We repeated this simulation many times and calculated the probability of occurrence of wave velocity.

The results are shown in Fig. 9 for $N=10,50,100$, and500. The fluctuation in wave velocity decreased as the number of oscillators increased. For example, the distribution had a $3 \sigma$ error of $5 \%$ for $N=100$ and of $1 \%$ for $N=500$. Therefore, putting a dummy zone consisting of many oscillators around the sensor will reduce the fluctuation in wave velocity. The output amplifier, shown in Fig. 8, can be used as the dummy zone, so no additional dummy zone will be needed in practice.

\section{References}

[1] Fujiwara A, Takahashi Y and Murase K 1997 Observation of single electron-hole recombination and photon-pumped current in an asymmetric Si single-electron transistor P. R. L.. 78 1532-35

[2] Nuryadi R, Ishikawa Y and Tabe M 2006 Single-photon-induced random telegraph signal in a two-dimensional multiple-tunnel-junction array Phys. Rev. B 73 45310-16

[3] Likharev K K 1999 Single-electron devices and their applications Proc. of IEEE 874 606-32

[4] Gravert H and Devoret M H 1992 Single Charge Tunneling-Coulomb Blockade Phenomena in Nanostructures New York: Plenum

[5] Oya T, Asai T, Fukui T and Amemiya Y 2005 Int. J. of Unconventional Computing 1 177-94

[6] Oya T, Asai T, Fukui T and Amemiya Y 2002 A majority-logic nanodevice using a balanced pair of single-electron boxes $J$. of Nanoscience and Nanotechnology2 333-42

[7] Kumakura K, Motohisa J and Fukui T 1997 Formation and characterization of coupled quantum dots (CQDs) by selective area metalorganic vapor phase epitaxy J. Crystal Growth 170 700-4

[8] Ong P H, Ang S S, Brown W D, Naseem H A, Ulrich R K and Dressendorfer P V 1990 Arsenicimplanted thin film PECVD semi-insulating polysilicon (SIPOS) resistors J. Ele. Mat. 20 211-5

[9] Kuwamura N, Taniguchi K and Hamaguchi C 1994 Simulation of single-electron logic circuits IEICE Trans. Electron. J77-C-II 221-8 